\title{
Is decompressive craniectomy useless in severe traumatic brain injury?
}

Junpeng Ma, Chao You*, Lu Ma and Siqing Huang

\begin{abstract}
Recently, a multicenter randomized controlled trial (RCT) by Cooper and colleagues indicated that decompressive craniectomy (DC) may be associated with a worse functional outcome in patients with diffuse traumatic brain injury (TBI), although DC can immediately and constantly reduce intracranial pressure (ICP). As this trial is well planned and of high quality, the unexpected result is meaningful. However, the evidence of the study is insufficient and the effect of DC in severe TBI is still uncertain. Additional multicenter RCTs are necessary to provide class I evidence on the role of DC in the treatment of refractory raised ICP after severe TBI.
\end{abstract}

Decompressive craniectomy (DC) is a straightforward procedure that for more than a century has been widely used to treat medically refractory intracranial hypertension of patients with severe traumatic brain injury (TBI). Although a series of clinical studies demonstrated that the procedure is the one of the most effective treatments in reducing intracranial pressure (ICP) $[1,2]$, no large prospective randomized controlled trial (RCT) had investigated the relation between successful or sustained reduction of increased ICP and functional outcomes after DC. An updated Cochrane review published in 2009 identified only one prospective randomized clinical trial ( $n=27$ participants) that evaluated the effect of DC in severe TBI [3]. The same year, a small ( $n=74$ patients) RCT that was published by Qiu and colleagues [4] indicated the beneficial effects of DC in patients with acute post-traumatic brain swelling.

In March of this year, a multicenter RCT by Cooper and colleagues [5] was published in the New England

*Correspondence: youchao028@163.com

Department of Neurosurgery, West China Hospital, Sichuan University,

37 Guoxuexiang Street, Chengdu 610041, The People's Republic of China
Journal of Medicine. Before this multicenter RCT, a pilot randomized trial [6] was completed to enable the multicenter DC study protocol. This multicenter RCT enrolled 155 adults with severe non-penetrating TBI and medically refractory intracranial hypertension from December 2002 through April 2010 but excluded patients with mass lesions. The results showed that, although DC can immediately and constantly reduce ICP (mean ICP of $14.4 \mathrm{~mm} \mathrm{Hg}$ versus $19.1 \mathrm{~mm} \mathrm{Hg} P<0.001$ ), the craniectomy group that received bifrontotemporoparietal DC $(\mathrm{n}=73)$ may be associated with a worse functional outcome than the standard-care group $(n=82)$ (odds ratio of 1.84 and $95 \%$ confidence interval of 1.05 to 3.24; $P=0.03)$. As this trial is well planned and of high quality, the unexpected result is meaningful and should be considered a reference for an evidence-based guideline. However, the evidence of the study is insufficient. First, the relatively small sample size is inadequate to provide a strong conclusion. Second, the thresholds for defining medically refractory intracranial hypertension (ICP of greater than $20 \mathrm{~mm} \mathrm{Hg}$ for more than 15 minutes within a 1-hour period after first-tier interventions) are not what many physicians would consider refractory. Third, in almost 3,500 potentially eligible patients, only 155 patients were enrolled (patients with a cerebral mass lesion were excluded). Therefore, the study cannot be generalized to all patients with severe non-penetrating brain injury. Fourth, after random assignment, more patients in the DC group had fixed and dilated pupils than patients in the medical therapy group (no reactivity of bilateral pupils: $27 \%$ versus $12 \%$ ), and this should be considered a potential risk of bias. Lastly, 15 patients (18\%) in the standard-care group underwent delayed DC as a lifesaving intervention. Although the investigators used intention-to-treat analysis, the bias introduced by the compassionate use of DC in the standard-care group should not be overlooked.

For these reasons, total disapproval of the effect of DC in severe TBI by some authors [7] is inappropriate. Additional multicenter RCTs are necessary to provide further conclusions on the efficacy of this procedure. In 2006, an international multicenter RCT comparing DC with medical management for refractory raised ICP was 
sponsored by the University of Cambridge [8]. This RESCUEicp (Randomized Evaluation of Surgery with Craniectomy for Uncontrollable Elevation of Intracranial Pressure) study planned to recruit 650 patients with refractory ICP after TBI (50 for the pilot phase and 600 for the main study) in an attempt to provide class I evidence on the role of surgical decompression in the treatment of raised ICP after severe TBI. Recently, an updated protocol [9] revealed that the RESCUEicp trial had recruited over 280 patients from more than 40 centers in 17 countries. We anxiously await the results of this international multicenter RCT and hope that they will enhance the evidence to guide the treatment of severe TBI.

\section{Abbreviations}

$D C$, decompressive craniectomy; ICP, intracranial pressure; $R C T$, randomized controlled trial; RESCUEicp, Randomized Evaluation of Surgery with

Craniectomy for Uncontrollable Elevation of Intracranial Pressure; TBI traumatic brain injury.

\section{Competing interests}

The authors declare that they have no competing interests.

\section{Published: 4 October 2011}

\section{References}

1. Whitfield PC, Patel H, Hutchinson PJ, Czosnyka M, Parry D, Menon D, Pickard $J D$, Kirkpatrick PJ: Bifrontal decompressive craniectomy in the management of posttraumatic intracranial hypertension. Br J Neurosurg 2001, 15:500-507.
2. Aarabi B, Hesdorffer DC, Ahn ES, Aresco C, Scalea TM, Eisenberg HM: Outcome following decompressive craniectomy for malignant swelling due to severe head injury. J Neurosurg 2006, 104:469-479.

3. Sahuquillo J: Decompressive craniectomy for the treatment of refractory high intracranial pressure in traumatic brain injury. Cochrane Database Syst Rev 2009, 2:CD003983.

4. Qiu W, Guo C, Shen H, Chen K, Wen L, Huang H, Ding M, Sun L, Jiang Q, Wang $W$ : Effects of unilateral decompressive craniectomy on patients with unilateral acute post-traumatic brain swelling after severe traumatic brain injury. Crit Care 2009, 13:R185.

5. Cooper DJ, Rosenfeld JV, Murray L, Arabi YM, Davies AR, D'Urso P, Kossmann T, Ponsford J, Seppelt I, Reilly P, Wolfe R: Decompressive craniectomy in diffuse traumatic brain injury. N Engl J Med 2011, 364:1493-1502.

6. Cooper DJ, Rosenfeld JV, Murray L, Wolfe R, Ponsford J, Davies A, D'Urso P, Pellegrino V, Malham G, Kossmann T: Early decompressive craniectomy for patients with severe traumatic brain injury and refractory intracranial hypertension--a pilot randomized trial. J Crit Care 2008, 23:387-393.

7. Marion DW: Decompressive craniectomy in diffuse traumatic brain injury. Lancet Neurol 2011, 10:497-498.

8. Hutchinson PJ, Corteen E, Czosnyka M, Mendelow AD, Menon DK, Mitchell P, Murray G, Pickard JD, Rickels E, Sahuquillo J, Servadei F, Teasdale GM, Timofeev I, Unterberg A, Kirkpatrick PJ: Decompressive craniectomy in traumatic brain injury: the randomized multicenter RESCUEicp study. Acta Neurochir Suppl 2006, 96:17-20.

9. Hutchinson PJ, Kolias AG, Timofeev I, Corteen E, Czosnyka M, Menon DK, Pickard JD, Kirkpatrick PJ: Update on the RESCUEicp decompressive craniectomy trial. Crit Care 2011, 15:P312.

doi:10.1186/cc10358

Cite this article as: Ma J, et al.: Is decompressive craniectomy useless in severe traumatic brain injury? Critical Care 2011, 15:193. 